\title{
Robust adaptive array beamforming under steering angle mismatch ${ }^{\text {is }}$
}

\author{
Ju-Hong Lee*, Kuang-Peng Cheng, Chih-Chang Wang \\ Room 517, Building 2, Graduate Institute of Communication Engineering, Department of Electrical Engineering, National Taiwan \\ University, Taipei 10617, Taiwan
}

Received 1 March 2004; received in revised form 8 February 2005; accepted 19 May 2005

Available online 5 July 2005

\begin{abstract}
The problem of adaptive array beamforming with multiple-beam constraints in the presence of steering angle error is considered. We first construct a cost function consisting of terms that utilize a posteriori information due to the received array data and an exponential constraint associated with steering angle error, respectively. Then, an appropriate estimate of the actual phase angle vector associated with each of the desired signals can be obtained by performing nonlinear optimization based on the cost function. An implementation algorithm is further presented to iteratively solve the problem. Theoretical analysis regarding the convergence property of the iterative procedure is also investigated. Finally, several computer simulation examples are provided for illustration and comparison.
\end{abstract}

(C) 2005 Elsevier B.V. All rights reserved.

Keywords: Adaptive array beamforming; Steering angle error

\section{Introduction}

An adaptive array beamformer is a spatial filter designed to extract the desired signal(s) while canceling interference and noise through spatial discrimination. The only a priori knowledge for a main-beam or a multiple-beam constrained beamformer is the actual direction vectors of the desired signals. The direction vector of a desired signal can be obtained from knowledge of the array sensor locations, signal directionsof-arrival, and propagation characteristics. However, the information may not be perfectly known in practice. This results in a mismatch between the presumed steering vectors and the actual direction vectors. Many reports have shown that the performance of a steered beam adaptive array beamformer is very sensitive to such mismatch [1-5].

\footnotetext{
This work was supported by the National Science Council of TAIWAN under Grant NSC92-2213-E002-033.

*Corresponding author. Fax: + 886223671909.

E-mail address: juhong@cc.ee.ntu.edu.tw (J.-H. Lee).
} 
To cure the problem of array performance degradation due to the above mismatch, a variety of robust techniques propose to impose additional constraints such as multiple linear constraints, derivative constraints, and norm constraints on the array weight vector [5-15]. However, imposing additional constraints deteriorates the array's capability to suppress interference and noise, i.e. it consumes some of the array's degrees-of-freedom. In contrast, the authors of [16] presented a robust approach based on the worst-case performance optimization for dealing with the problem of array performance degradation due to the signal covariance matrix with some fixed error. On the other hand, the problem of the steering vector error due to signal look direction mismatch is considered in [17] to formulate a constrained minimization problem which can be solved by convex optimization-based implementation using second-order cone programming. However, this method provides array performance very similar to that of using the simple so-called diagonal loading of the sample matrix inversion (LSMI) algorithm [18] and comparable with that of using eigenspace-based approaches [19] when the signal-to-noise ratio (SNR) is reasonably high. Recently, the authors of [20] have considered the beamforming problem when the desired signal has a nonrandom steering vector error using the Capon beamformer. A robust method for determining the diagonal loading value is also proposed. All of the above-mentioned techniques [5-20] are developed in the case of adaptive beamforming with main-beam constraint. In many applications, such as satellite communications [21], an antenna array must possess beamforming capability to receive more than one signal with specified gain requirements while suppressing all jammers. This purpose can be effectively achieved by using an antenna array with multiple-beam pattern [21,22]. Recently, a technique for adaptive beamforming with the capability of providing multiple-beam constraints (MBC) has been presented in [23].

In this paper, the problem of adaptive beamforming with $\mathrm{MBC}$ in the presence of steering angle error is considered. Instead of directly dealing with the estimation of the actual steering angle, we handle an equivalent problem where the corresponding steering phase angle vector is to be found. A robust method in conjunction with an iterative procedure is presented for coping with the considered problem. To find the optimal phase angle vector, we construct a cost function consisting of the squared norm of the projection of the steering vector on the noise subspace and a constraint related to an exponential function of the squared norm of the resulting phase error vector. The proposed cost function uses an exponential constraint instead of a non-exponential constraint proposed by a recent research work [24] which deals only with main-beam adaptive beamforming. Minimizing the squared norm of the projection of the steering vector on the noise subspace is equivalent to maximizing the squared norm of the projection of the steering vector on the signal plus interference subspace. The constraint related to an exponential function of the squared norm of the resulting phase error vector is utilized to prevent the obtained optimal phase angle vector for each desired signal from becoming one of the interference phase angle vectors. Since the resulting minimization problem is highly nonlinear, we use a gradient method to iteratively find the solution. It is shown that using the exponential constraint provides the advantage of properly adjusting the step size during the gradient search procedure. The analysis regarding the investigation of the convergence property of the proposed method is also presented. Several computer simulation examples show the effectiveness of the proposed method.

This paper is organized as follows. Section 2 formulates the problem of adaptive beamforming with MBC in the presence of steering angle error. Then, a robust method is presented in Section 3 for dealing with the considered problem. In Section 4, we present a theoretical analysis to provide a proof regarding the convergence property of the proposed method. Section 5 shows several simulation examples to illustrate the effectiveness of the proposed method. Finally, we conclude the paper in Section 6.

\section{Problem formulation}

Consider a uniform linear array (ULA) with $M$ sensors and interelement spacing equal to half of the smallest wavelength of the signals. Let $K$ uncorrelated narrow-band and far-field signals impinge on the 
array from direction angles $\theta_{i}, i=1,2, \ldots, K$, with respect to array broadside. The signal received at the $m$ th array sensor can be expressed as

$$
x_{m}(t)=\sum_{i=1}^{K} s_{i}(t) a_{m}\left(\theta_{i}\right)+n_{m}(t), \quad m=1,2, \ldots, M,
$$

where $a_{m}\left(\theta_{i}\right)=\exp \left(j\left(2 \pi d_{m} \sin \theta_{i}\right) / \lambda_{i}\right), \lambda_{i}$ is the wavelength of the $i$ th signal, and $d_{m}$ is the distance between the $m$ th and the first array sensors, $s_{i}(t)$ is the complex waveform of the $i$ th signal, and $n_{m}(t)$ is the spatially white noise with mean zero and variance $\sigma_{n}^{2}$ received at the $m$ th array sensor. In matrix form, we can write the data vector received by the ULA as follows:

$$
\boldsymbol{x}(t)=\boldsymbol{B} \boldsymbol{s}(t)+\boldsymbol{n}(t),
$$

where the matrix $B=\left[\boldsymbol{a}\left(\theta_{1}\right) \boldsymbol{a}\left(\theta_{2}\right) \ldots \boldsymbol{a}\left(\theta_{K}\right)\right]$ with the direction vector of the $i$ th signal being given by $\boldsymbol{a}\left(\theta_{i}\right)=\left[a_{1}\left(\theta_{i}\right) a_{2}\left(\theta_{i}\right) \ldots a_{M}\left(\theta_{i}\right)\right]^{\mathrm{T}}$, the signal source vector is $\boldsymbol{s}(t)=\left[s_{1}(t) s_{2}(t) \cdots s_{K}(t)\right]^{\mathrm{T}}$, and the noise vector is $\boldsymbol{n}(t)=\left[n_{1}(t) n_{2}(t) \ldots n_{M}(t)\right]^{\mathrm{T}}$. The superscript $\mathrm{T}$ denotes the transpose operation. Under the assumption that $s(t)$ and $n(t)$ are uncorrelated, the $M \times M$ ensemble correlation matrix of $x(t)$ is Toeplitz-Hermitian and given by

$$
\boldsymbol{R}_{x}=\left[R_{k l}\right]=[R(k-l)]=\mathrm{E}\left\{\boldsymbol{x}(t) \boldsymbol{x}(t)^{\mathrm{H}}\right\}=\boldsymbol{B} \boldsymbol{R}_{s} \boldsymbol{B}^{\mathrm{H}}+\sigma_{n}^{2} \boldsymbol{I},
$$

where the superscript $\mathrm{H}$ denotes the complex conjugate transpose. $\boldsymbol{R}_{s}=\mathrm{E}\left\{\boldsymbol{s}(t) \boldsymbol{s}(t)^{\mathrm{H}}\right\}$ has rank $K$ if the $K$ signals are uncorrelated.

Let the ULA use a weight vector $\boldsymbol{w}=\left[w_{1} w_{2} \ldots w_{M}\right]$ for processing the received data vector $\boldsymbol{x}(t)$ to produce the array output signal $y(t)=\boldsymbol{w}^{\mathrm{H}} \boldsymbol{x}(t)$. Assume that the selective gain/null requirements are specified by assigning a gain $c_{p}$ at the direction vector $\boldsymbol{a}\left(\theta_{p}\right)$ for $p=1,2, \ldots, P$, where $P$ denotes the number of signals with gain/null constraint. Then, the optimal weight vector for the adaptive array can be found from the following constrained optimization problem [21]:

$$
\begin{aligned}
& \text { Minimize } \mathrm{E}\left\{|y(t)|^{2}\right\}=\boldsymbol{w}^{\mathrm{H}} \boldsymbol{R}_{x} \boldsymbol{w} \\
& \text { Subject to } \boldsymbol{G}^{\mathrm{H}} \boldsymbol{w}=\boldsymbol{c},
\end{aligned}
$$

where the matrix $\boldsymbol{G}=\left[\boldsymbol{a}\left(\theta_{1}\right) \boldsymbol{a}\left(\theta_{2}\right) \cdots \boldsymbol{a}\left(\theta_{P}\right)\right]$ denotes the constraint matrix and $\boldsymbol{c}=\left[c_{1} c_{2} \ldots c_{P}\right]$ denotes the gain vector. Accordingly, the optimal weight vector is given by

$$
\boldsymbol{w}_{o}=\boldsymbol{R}_{x}^{-1} \boldsymbol{G}\left(\boldsymbol{G}^{\mathrm{H}} \boldsymbol{R}_{x}^{-1} \boldsymbol{G}\right)^{-1} \boldsymbol{c} .
$$

Substituting (5) into $\mathrm{E}\left\{|y(t)|^{2}\right\}=\boldsymbol{w}^{\mathrm{H}} \boldsymbol{R}_{x} \boldsymbol{w}$ yields the corresponding array output power equal to

$$
\mathrm{E}\left\{|y(t)|^{2}\right\}=\boldsymbol{w}^{\mathrm{H}} \boldsymbol{R}_{x} \boldsymbol{w}=\boldsymbol{c}^{\mathrm{H}}\left(\boldsymbol{G}^{\mathrm{H}} \boldsymbol{R}_{x}^{-1} \boldsymbol{G}\right)^{-1} \boldsymbol{c} .
$$

In the presence of a steering angle error, let the error vector associated with the direction angles be $\boldsymbol{\theta}_{e}=\left[\Delta \theta_{1} \Delta \theta_{2} \ldots \Delta \theta_{P}\right]^{\mathrm{T}}$. We consider that the phase angle error vector for the signal with gain $c_{p}$ due to the direction angle error $\Delta \theta_{p}, p=1,2, \ldots, P$, is given by

$$
\boldsymbol{\Theta}_{e p}=\boldsymbol{\Theta}_{p}-\boldsymbol{\Theta}_{d p},
$$

where $\boldsymbol{\Theta}_{p}$ and $\boldsymbol{\Theta}_{d p}$ denote the phase angle vectors associated with actual direction vector $\boldsymbol{a}\left(\theta_{p}\right)$ and the presumed direction vector $\boldsymbol{a}_{d}\left(\theta_{p}\right)$, respectively. Without loss of generality, let the $m$ th entry of the actual direction vector $\boldsymbol{a}\left(\theta_{p}\right)$ be expressed as $a_{m}\left(\theta_{p}\right)=\exp \left(j v_{p m}\right)$ and the corresponding phase angle vector be constructed as $\boldsymbol{\Theta}_{p}=\left[\begin{array}{llll}v_{p 1} & v_{p 2} & \ldots & v_{p M}\end{array}\right]^{\mathrm{T}}$. Similarly, let the $m$ th entry of the presumed direction vector $\boldsymbol{a}_{d}\left(\theta_{p}\right)$ be expressed as $a_{d m}\left(\theta_{p}\right)=\exp \left(j v_{d p m}\right)$ and the corresponding phase angle vector be constructed as $\boldsymbol{\Theta}_{d p}=\left[\begin{array}{llll}v_{d p} 1 & v_{d p 2} & \ldots & v_{d p M}\end{array}\right]^{\mathrm{T}}$. We finally construct an $M \times P$ phase angle error matrix $\boldsymbol{\Psi}$ and the 
corresponding $P M \times 1$ phase angle error vector $\Gamma$ for the $P$ uncorrelated signals as follows:

$$
\begin{aligned}
& \boldsymbol{\Psi}=\left[\boldsymbol{\Theta}_{e 1} \boldsymbol{\Theta}_{e 2} \ldots \boldsymbol{\Theta}_{e P}\right], \\
& \boldsymbol{\Gamma}=\left[\boldsymbol{\Theta}_{e 1}^{\mathrm{T}} \boldsymbol{\Theta}_{e 2}^{\mathrm{T}} \ldots \boldsymbol{\Theta}_{e P}^{\mathrm{T}}\right] .
\end{aligned}
$$

To deal with the problem of array beamforming with $\mathrm{MBC}$ in the presence of steering angle errors as described above, we present a robust method in the next section.

\section{A robust method}

From the property of a gain-constrained array beamformer, it is well known that the output power of the beamformer will achieve its maximum when each presumed direction vector $\boldsymbol{a}_{d}\left(\theta_{p}\right)$ of the constraint matrix $\boldsymbol{G}$ coincides with the actual direction vector $\boldsymbol{a}\left(\theta_{p}\right), p=1,2, \ldots, P$. Moreover, from the eigendecomposition of $\boldsymbol{R}_{x}$, we can express $\boldsymbol{R}_{x}=\sum_{i=1}^{M} \lambda_{i} \boldsymbol{e}_{i} \boldsymbol{e}_{i}^{\mathrm{H}}$, where $\lambda_{1} \geqslant \lambda_{2} \geqslant \ldots \geqslant \lambda_{J+P} \geqslant \lambda_{J+P+1}=\ldots=\lambda_{M}=\sigma_{n}^{2}$, are the eigenvalues of $\boldsymbol{R}_{x}$ in the descending order, $\boldsymbol{e}_{i}$ are the corresponding eigenvectors, and $J$ is the number of interferers. The eigenvectors associated with the minimum eigenvalue $\sigma_{n}^{2}$ are orthogonal to the direction vectors of the signals with specified gain/null constraints and interferers. Therefore, the subspaces spanned by $\boldsymbol{E}_{n}=\left\{\boldsymbol{e}_{J+P+1}, \ldots, \boldsymbol{e}_{M}\right\}$ (called the noise subspace) and $\boldsymbol{E}_{s}=\left\{\boldsymbol{e}_{1}, \boldsymbol{e}_{2}, \ldots, \boldsymbol{e}_{J+P}\right\}$ (called the signal plus interference subspace) are orthogonal. Consequently, we can rewrite $\boldsymbol{R}_{x}$ as follows:

$$
\boldsymbol{R}_{x}=\sum_{i=1}^{M} \lambda_{i} \boldsymbol{e}_{i} \boldsymbol{e}_{i}^{\mathrm{H}}=\boldsymbol{E}_{s} \boldsymbol{\Lambda}_{s} \boldsymbol{E}_{s}^{\mathrm{H}}+\boldsymbol{E}_{n} \boldsymbol{\Lambda}_{n} \boldsymbol{E}_{n}^{\mathrm{H}},
$$

where $\boldsymbol{\Lambda}_{s}=\operatorname{diag}\left\{\lambda_{1}, \lambda_{2}, \ldots, \lambda_{J+P}\right\}$ and $\boldsymbol{\Lambda}_{n}=\sigma_{n}^{2} \boldsymbol{I}$, where $\boldsymbol{I}$ denotes the identity matrix with appropriate size. Based on (6) and (10), we create an appropriate cost function regarding the phase angle errors as

$$
J(\boldsymbol{\Phi})=\sum_{p=1}^{P}\left(\boldsymbol{s}_{p}\right)^{\mathrm{H}} \boldsymbol{E}_{n} \boldsymbol{E}_{n}^{\mathrm{H}}\left(\boldsymbol{s}_{p}\right)-\kappa \exp \left\{-\sum_{p=1}^{P}\left[\left(\boldsymbol{\Theta}_{s p}-\boldsymbol{\Theta}_{d p}\right)^{\mathrm{T}}\left(\boldsymbol{\Theta}_{s p}-\boldsymbol{\Theta}_{d p}\right)\right] / 2\right\},
$$

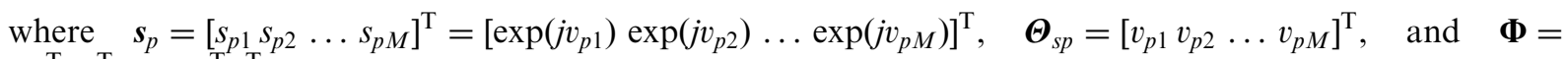
$\left[\begin{array}{llll}\boldsymbol{\Theta}_{s 1}^{\mathrm{T}} & \boldsymbol{\Theta}_{s 2}^{\mathrm{T}} & \ldots & \boldsymbol{\Theta}_{s P}^{\mathrm{T}}\end{array}\right]^{\mathrm{T}}$. The first term of (11) represents the squared norm of the projection of the constraint vectors $\boldsymbol{s}_{p}, p=1,2, \ldots, P$, on the noise subspace spanned by $\boldsymbol{E}_{n}$; the second term is the constraint related to the squared norm of the phase angle error, and $\kappa$ denotes a positive weighting parameter providing the relative weight between these terms. According to the theoretical analysis presented in [25], we would expect that the proposed method shows better capabilities against the finite sample effect because of using a projection scheme to estimate the phase angle vector as shown by the first term of Eq. (11).

As a result, the optimal solution $\boldsymbol{\Phi}_{o}$ in minimizing (11) can then be used as an appropriate estimate of $\boldsymbol{\Phi}_{P}=\left[\boldsymbol{\Theta}_{1}^{\mathrm{T}} \boldsymbol{\Theta}_{2}^{\mathrm{T}} \ldots \boldsymbol{\Theta}_{P}^{\mathrm{T}}\right]^{\mathrm{T}}$ formed by the actual phase angle vectors $\boldsymbol{\Theta}_{p}, p=1,2, \ldots, P$, for array beamforming. However, the cost function of (11) is a highly nonlinear function of the phase angle vectors $\boldsymbol{\Theta}_{p}, p=1,2, \ldots, P$. Thus, a closed-form solution for the optimal solution cannot exist. We resort to an iterative procedure to solve this problem as follows. First, we rewrite (11) as follows:

$$
J(\boldsymbol{\Phi})=(\mathbf{S})^{\mathrm{H}} \mathbf{W}(\mathbf{S})-\kappa \exp \left\{-\sum_{p=1}^{P}\left[\left(\boldsymbol{\Theta}_{s p}-\boldsymbol{\Theta}_{d p}\right)^{\mathrm{T}}\left(\boldsymbol{\Theta}_{s p}-\boldsymbol{\Theta}_{d p}\right)\right] / 2\right\},
$$

where the $M P \times 1$ vector $\mathbf{S}=\left[s_{1}^{\mathrm{T}} s_{2}^{\mathrm{T}} \ldots s_{P}^{\mathrm{T}}\right]^{\mathrm{T}}$ and $\mathbf{W}$ is a $P M \times P M$ block diagonal matrix with the $p$ th $M \times M$ diagonal block matrix given by $\boldsymbol{E}_{n} \boldsymbol{E}_{n}{ }^{\mathrm{H}}$. Then, the gradient vector of $J(\boldsymbol{\Phi})$ can be computed 
according to

$$
\begin{aligned}
\nabla_{\Phi} J(\boldsymbol{\Phi})= & {\left[-2 \operatorname{Re}\left\{j\left\{\left(\boldsymbol{E}_{n} \boldsymbol{E}_{n}^{\mathrm{H}} \boldsymbol{s}_{1}\right) \odot \boldsymbol{s}_{1}^{*}\right\}\right\}-2 \operatorname{Re}\left\{j\left\{\left(\boldsymbol{E}_{n} \boldsymbol{E}_{n}^{\mathrm{H}} \boldsymbol{s}_{2}\right) \odot \boldsymbol{s}_{2}^{*}\right\}\right\} \cdots-2 \operatorname{Re}\left\{j\left\{\left(\boldsymbol{E}_{n} \boldsymbol{E}_{n}^{\mathrm{H}} \boldsymbol{s}_{P}\right) \odot \boldsymbol{s}_{P}^{*}\right\}\right\}\right]^{\mathrm{T}} } \\
& +\kappa\left[\exp \left\{-\sum_{p=1}^{P}\left[\left(\boldsymbol{\Theta}_{s p}-\boldsymbol{\Theta}_{d p}\right)^{\mathrm{T}}\left(\boldsymbol{\Theta}_{s p}-\boldsymbol{\Theta}_{d p}\right)\right] / 2\right\}\left(\boldsymbol{\Phi}-\boldsymbol{\Phi}_{d}\right)\right]
\end{aligned}
$$

where $\operatorname{Re}\{x\}$ denotes the real part of $x$, the superscript $*$ the complex conjugate, and $\odot$ the Hadamard (or elementwise) product [26]. $\boldsymbol{\Phi}_{d}=\left[\boldsymbol{\Theta}_{d 1}^{\mathrm{T}} \boldsymbol{\Theta}_{d 2}^{\mathrm{T}} \ldots \Theta_{d P}^{\mathrm{T}}\right]^{\mathrm{T}}$. Then, we update the phase angle vector $\boldsymbol{\Phi}$ and the corresponding steering constraint vector $\boldsymbol{s}_{p}$ as follows:

$$
\begin{aligned}
& \boldsymbol{\Phi}^{(k+1)}=\left[\left(\boldsymbol{\Theta}_{s 1}^{(k+1)}\right)^{\mathrm{T}}\left(\boldsymbol{\Theta}_{s 2}^{(k+1)}\right)^{\mathrm{T}} \ldots\left(\boldsymbol{\Theta}_{s P}^{(k+1)}\right)^{\mathrm{T}}\right]^{\mathrm{T}}=\boldsymbol{\Phi}^{(k)}-\varepsilon \nabla_{\Phi} J\left(\boldsymbol{\Phi}^{(k)}\right), \\
& s_{p m}^{(k+1)}=\exp \left(j v_{p m}^{(k+1)}\right), \quad p=1,2, \ldots, P, m=1,2, \ldots, M,
\end{aligned}
$$

where the superscript $k$ in bracket denotes the $k$ th iteration and $\varepsilon$ the preset positive step size. From (14), we note that the second term includes the factor of the squared norm related to each of the phase angle vector errors $\boldsymbol{\Theta}_{s p}-\boldsymbol{\Theta}_{d p}, p=1,2, \ldots, P$, at the $k$ th iteration. Hence, it would be expected that the resulting gradient approach for finding the optimal $\boldsymbol{\Phi}$ can provide a more appropriate estimate of $\boldsymbol{\Phi}$ since the resulting step size becomes variable due to the exponential term as shown in (13).

Next, we present an appropriate scheme for choosing the initial estimate for the $M P \times 1$ vector $\mathbf{S}=$ $\left[s_{1}^{\mathrm{T}} s_{2}^{\mathrm{T}} \ldots s_{P}^{\mathrm{T}}\right]^{\mathrm{T}}$ in order to initiate the iterative process of the proposed robust method. According to the optimal weight vector given by (5) under the assumption that $P=1$ and the desired signal with direction vector $\boldsymbol{a}\left(\theta_{p}\right)$, the output of the adaptive array is approximately given by

$$
y_{p}(t)=\boldsymbol{w}_{o p}^{\mathrm{H}} \boldsymbol{x}(t) \approx s_{p}(t) g_{p}+\boldsymbol{w}_{o p}^{\mathrm{H}} \boldsymbol{n}(t)
$$

based on the assumptions that $M>K$ and the interference signals are suppressed enough, where $g_{p} \equiv$ $\boldsymbol{w}_{o p}^{\mathrm{H}} \boldsymbol{a}\left(\theta_{p}\right)$ denotes the array gain for the specified signal, where subscript $p$ represents the results obtained by using the desired signal with direction vector $\boldsymbol{a}\left(\theta_{p}\right), p=1,2, \ldots, P$. Eq. (16) reveals that the output of the adaptive array can be used as a reference signal to find the actual phase angle vector $\boldsymbol{\Theta}_{p}$. Consider the crosscorrelation between $\boldsymbol{x}(t)$ and $\mathrm{y}_{p}(t)$. We have

$$
\mathrm{E}\left\{\boldsymbol{x}(t) y_{p}(t)^{*}\right\}=\mathrm{E}\left\{\boldsymbol{x}(t) \boldsymbol{x}(t)^{\mathrm{H}}\right\} \boldsymbol{w}_{o p}=\boldsymbol{R}_{x} \boldsymbol{w}_{o p} \approx \pi_{p} g_{p}^{*} \boldsymbol{a}\left(\theta_{p}\right)+\sigma_{n}^{2} \boldsymbol{w}_{o p},
$$

where $\pi_{p}$ denotes the power associated with the specified signal. In practice, the noise power $\sigma_{n}{ }^{2}$ is unknown. However, it can be estimated by taking the average of the $M-K$ smallest eigenvalues of the autocorrelation matrix as the estimate of $\sigma_{n}^{2}$. This raises the issue of estimating the number $K$ of signals/ jammers, which is in itself a difficult signal processing problem. Nevertheless, the techniques based on AIC/ MDL criteria proposed by Wax and Kailath [27] and Fiscler et al. [28] or the bootstrap-based technique proposed by Brcich et al. [29] can be utilized to deal with this difficulty. From (17), we can therefore adopt the following vector as the initial estimates for each of $s_{p}$ :

$$
\boldsymbol{v}_{p}=\boldsymbol{R}_{x} \boldsymbol{w}_{o p}-\sigma_{n}^{2} \boldsymbol{w}_{o p} .
$$

From (18), we note that the direction vector $\boldsymbol{a}\left(\theta_{p}\right)$ is approximately proportional to $\boldsymbol{v}_{p}$ with a proportional constant $\pi_{p} g_{p}^{*}$. Hence, an appropriate initial estimate $\boldsymbol{s}_{p}{ }^{(0)}$ for $\boldsymbol{s}_{p}$ can be formed as follows:

$$
\boldsymbol{u}_{p}=\left[u_{p 1}, u_{p 2}, \ldots, u_{p M}\right]^{\mathrm{T}}=\left(v_{p 1}\right)^{-1} \boldsymbol{v}_{p}, \quad s_{p m}^{(0)}=\left|u_{p m}\right|^{-1} u_{p m}, \quad m=1,2, \ldots, M,
$$




$$
\boldsymbol{s}_{p}^{(0)}=\left[s_{p 1}^{(0)}, s_{p 2}^{(0)}, \ldots, s_{p M}^{(0)}\right]^{\mathrm{T}}
$$

for $p=1,2, \ldots, P$, where $v_{p 1}$ denotes the first entry of $\boldsymbol{v}_{p}$. The superscript " 0 " in bracket identifies the initial estimate. In other words, we keep only the phase portion of $\boldsymbol{v}_{p}$ and then take the phase referencing to the first element of $\boldsymbol{v}_{p}$ to form the initial estimate $\boldsymbol{s}_{p}{ }^{(0)}$. Finally, we construct an initial estimate $\mathbf{S}^{(0)}=$ $\left[\left(s_{1}^{(0)}\right)^{\mathrm{T}}\left(s_{2}^{(0)}\right)^{\mathrm{T}} \ldots\left(s_{P}^{(0)}\right)^{\mathrm{T}}\right]^{\mathrm{T}}$ of $\mathbf{S}$ for carrying out the proposed iterative process.

\section{Convergence of the proposed method}

In this section, the convergence property of the proposed method is evaluated. To ensure convergence, we have to show that the cost function to be minimized as given by (12) possesses the property of $J\left(\boldsymbol{\Phi}^{(k+1)}\right)<J\left(\boldsymbol{\Phi}^{(k)}\right)$. For the sake of simplicity, we let the vector $\boldsymbol{A}^{(k)}$ represent the second term of (14), i.e., $\quad \boldsymbol{A}^{(k)}=-\varepsilon \nabla_{\phi} J\left(\boldsymbol{\Phi}^{(k)}\right)=\left[\left(\boldsymbol{A}_{1}^{(k)}\right)^{\mathrm{T}}\left(\boldsymbol{A}_{2}^{(k)}\right)^{\mathrm{T}} \ldots\left(\boldsymbol{A}_{P}^{(k)}\right)^{\mathrm{T}}\right]^{\mathrm{T}} \quad$ and $\quad \boldsymbol{A}_{p}^{(k)}=\left[A_{p 1}^{(k)} A_{p 2}^{(k)} \ldots A_{p M}^{(k)}\right]^{\mathrm{T}}, \quad p=1,2, \ldots, P$. Assume that $\boldsymbol{A}^{(\mathrm{k})}$ is a nonzero real vector with a norm small enough at the $k$ th iteration. Then, $\left(\boldsymbol{A}^{(k)}\right)^{\mathrm{T}} \boldsymbol{A}^{(k)}>0$, i.e.,

$$
\left(\boldsymbol{A}^{(k)}\right)^{\mathrm{T}}\left\{2 \varepsilon \operatorname{Re}\left\{j\left[\mathbf{W}\left(\mathbf{S}^{(k)}\right)\right] \odot\left(\mathbf{S}^{(k)}\right)^{*}\right\}-\varepsilon \kappa \exp \left\{-\left[\left(\boldsymbol{\Phi}^{(k)}-\boldsymbol{\Phi}_{d}\right)^{\mathrm{T}}\left(\boldsymbol{\Phi}^{(k)}-\boldsymbol{\Phi}_{d}\right)\right] / 2\right\}\left(\boldsymbol{\Phi}^{(k)}-\boldsymbol{\Phi}_{d}\right)\right\}>0 .
$$

Hence,

$$
\left(\boldsymbol{A}^{(k)}\right)^{\mathrm{T}}\left\{2 \operatorname{Re}\left\{j\left[\mathbf{W}\left(\mathbf{S}^{(k)}\right)\right] \odot\left(\mathbf{S}^{(k)}\right)^{*}\right\}\right\}>\kappa \exp \left\{-\left[\left(\boldsymbol{\Phi}^{(k)}-\boldsymbol{\Phi}_{d}\right)^{\mathrm{T}}\left(\boldsymbol{\Phi}^{(k)}-\boldsymbol{\Phi}_{d}\right)\right] / 2\right\}\left(\boldsymbol{A}^{(k)}\right)^{\mathrm{T}}\left(\boldsymbol{\Phi}^{(k)}-\boldsymbol{\Phi}_{d}\right)
$$

and the objective function after the $(k+1)$ th iteration is given by

$$
J\left(\boldsymbol{\Phi}^{(k+1)}\right)=\left(\mathbf{S}^{(k+1)}\right)^{\mathrm{H}} \mathbf{W}\left(\mathbf{S}^{(k+1)}\right)-\kappa \exp \left\{-\left[\left(\boldsymbol{\Phi}^{(k+1)}-\boldsymbol{\Phi}_{d}\right)^{\mathrm{T}}\left(\boldsymbol{\Phi}^{(k+1)}-\boldsymbol{\Phi}_{d}\right)\right] / 2\right\} .
$$

According to the above definition, (14), and (15), we have

$$
\begin{gathered}
s_{p m}^{(k+1)}=\exp \left(j v_{p m}^{(k+1)}\right)=\exp \left\{j\left(v_{p m}^{(k)}+A_{p m}^{(k)}\right)\right\} \approx\left(1+j A_{p m}^{(k)}\right) \exp \left(j v_{p m}^{(k)}\right), \\
p=1,2, \ldots, P, \quad m=1,2, \ldots, M .
\end{gathered}
$$

Based on the result of (23) and the definition of (20), we can express the $M P \times 1$ vector $\mathbf{S}$ after the $(k+1)$ th iteration as follows:

$$
\mathbf{S}^{(k+1)} \approx \mathbf{S}^{(k)}+\left(j \boldsymbol{A}^{(k)}\right) \odot \mathbf{S}^{(k)}
$$

Substituting (24) into (22) and performing the necessary algebraic manipulations yields

$$
\begin{aligned}
J\left(\boldsymbol{\Phi}^{(k+1)}\right) \approx & {\left[\mathbf{S}^{(k)}+\left(j \boldsymbol{A}^{(k)}\right) \odot \mathbf{S}^{(k)}\right]^{\mathrm{H}} \mathbf{W}\left[\mathbf{S}^{(k)}+\left(j \boldsymbol{A}^{(k)}\right) \odot \mathbf{S}^{(k)}\right] } \\
& -\kappa \exp \left\{-\left[\left(\boldsymbol{\Phi}^{(k)}+\boldsymbol{A}^{(k)}-\mathbf{\Phi}_{d}\right)^{\mathrm{T}}\left(\boldsymbol{\Phi}^{(k)}+\boldsymbol{A}^{(k)}-\mathbf{\Phi}_{d}\right)\right] / 2\right\} \\
\approx & \left(\mathbf{S}^{(k)}\right)^{\mathrm{H}} \mathbf{W}\left(\mathbf{S}^{(k)}\right)-\kappa \exp \left\{-\left[\left(\boldsymbol{\Phi}^{(k)}-\boldsymbol{\Phi}_{d}\right)^{\mathrm{T}}\left(\boldsymbol{\Phi}^{(k)}-\boldsymbol{\Phi}_{d}\right)\right] / 2\right\}+\left(\mathbf{S}^{(k)}\right)^{\mathrm{H}} \odot\left[-j \boldsymbol{A}^{(k)}\right] \mathbf{W}\left(\mathbf{S}^{(k)}\right)
\end{aligned}
$$




$$
\begin{aligned}
& +\left(\mathbf{S}^{(k)}\right)^{\mathrm{H}} \mathbf{W}\left[j \boldsymbol{A}^{(k)}\right] \odot\left(\mathbf{S}^{(k)}\right)+\left(\mathbf{S}^{(k)}\right)^{\mathrm{H}} \odot\left[-j \boldsymbol{A}^{(k)}\right] \mathbf{W}\left[j \boldsymbol{A}^{(k)}\right] \odot\left(\mathbf{S}^{(k)}\right) \\
& +\left\{\left(\boldsymbol{\Phi}^{(k)}-\mathbf{\Phi}_{d}\right)^{\mathrm{T}} \boldsymbol{A}^{(k)}+\left(\boldsymbol{A}^{(k)}\right)^{\mathrm{T}} \boldsymbol{A}^{(k)} / 2\right\} \kappa \exp \left\{-\left[\left(\boldsymbol{\Phi}^{(k)}-\mathbf{\Phi}_{d}\right)^{\mathrm{T}}\left(\boldsymbol{\Phi}^{(k)}-\mathbf{\Phi}_{d}\right)\right] / 2\right\} \\
\approx & J\left(\boldsymbol{\Phi}^{(k)}\right)+2 \operatorname{Re}\left\{\left(\mathbf{S}^{(k)}\right)^{\mathrm{H}} \mathbf{W}\left[j \boldsymbol{A}^{(k)}\right] \odot\left(\mathbf{S}^{(k)}\right)\right\} \\
& +\left(\boldsymbol{\Phi}^{(k)}-\mathbf{\Phi}_{d}\right)^{\mathrm{T}} \boldsymbol{A}^{(k)} \kappa \exp \left\{-\left[\left(\boldsymbol{\Phi}^{(k)}-\mathbf{\Phi}_{d}\right)^{\mathrm{T}}\left(\boldsymbol{\Phi}^{(k)}-\mathbf{\Phi}_{d}\right)\right] / 2\right\}
\end{aligned}
$$

since the norm of $\boldsymbol{A}^{(k)}$ is small enough, we neglect the terms $\left(\boldsymbol{A}^{(k)}\right)^{\mathrm{T}} \boldsymbol{A}^{(k)}$ and $\left(\mathbf{S}^{(k)}\right)^{\mathrm{H}} \odot\left[-j \boldsymbol{A}^{(k)}\right] \mathbf{W}\left[j \boldsymbol{A}^{(k)}\right] \odot\left(\mathbf{S}^{(k)}\right)$. From (25), it is clear that we have to show

$$
2 \operatorname{Re}\left\{\left(\mathbf{S}^{(k)}\right)^{\mathrm{H}} \mathbf{W}\left[j \boldsymbol{A}^{(k)}\right] \odot\left(\mathbf{S}^{(k)}\right)\right\}+\left(\boldsymbol{\Phi}^{(k)}-\boldsymbol{\Phi}_{d}\right)^{\mathrm{T}} \boldsymbol{A}^{(k)} \kappa \exp \left\{-\left[\left(\boldsymbol{\Phi}^{(k)}-\boldsymbol{\Phi}_{d}\right)^{\mathrm{T}}\left(\boldsymbol{\Phi}^{(k)}-\boldsymbol{\Phi}_{d}\right)\right] / 2\right\} \leqslant 0
$$

for any $k$ in order to ensure convergence. Based on (21), condition (26) can be reformulated as follows:

$$
2 \operatorname{Re}\left\{\left(\mathbf{S}^{(k)}\right)^{\mathrm{H}} \mathbf{W}\left[j \boldsymbol{A}^{(k)}\right] \odot\left(\mathbf{S}^{(k)}\right)\right\}+\left(\boldsymbol{A}^{(k)}\right)^{\mathrm{T}} 2 \operatorname{Re}\left\{j\left[\mathbf{W} \mathbf{S}^{(k)}\right] \odot\left(\mathbf{S}^{(k)}\right)^{*}\right\} \leqslant 0
$$

which can be reformulated as follows:

$$
\sum_{p=1}^{P} 2 \operatorname{Re}\left\{\left(\mathbf{S}_{p}^{(k)}\right)^{\mathrm{H}} \boldsymbol{E}_{n} \boldsymbol{E}_{n}^{\mathrm{H}}\left[j \boldsymbol{A}_{p}^{(k)}\right] \odot\left(\mathbf{S}_{p}^{(k)}\right)\right\}+\sum_{p=1}^{P}\left(\boldsymbol{A}^{(k)}\right)^{\mathrm{T}} 2 \operatorname{Re}\left\{\left[j \boldsymbol{E}_{n} \boldsymbol{E}_{n}^{\mathrm{H}} \mathbf{S}_{p}^{(k)}\right] \odot\left(\mathbf{S}_{p}^{(k)}\right)^{*}\right\} \leqslant 0 .
$$

Next, we manipulate the left-hand side of (28) as follows:

$$
\begin{array}{rl}
\sum_{p=1}^{P} & 2 \operatorname{Re}\left\{\left(\mathbf{S}_{p}^{(k)}\right)^{\mathrm{H}} \boldsymbol{E}_{n} \boldsymbol{E}_{n}^{\mathrm{H}}\left[j \boldsymbol{A}_{p}^{(k)}\right] \odot\left(\mathbf{S}_{p}^{(k)}\right)\right\}+\sum_{p=1}^{P}\left(\boldsymbol{A}^{(k)}\right)^{\mathrm{T}} 2 \operatorname{Re}\left\{\left[j \boldsymbol{E}_{n} \boldsymbol{E}_{n}^{\mathrm{H}} \mathbf{S}_{p}^{(k)}\right] \odot\left(\mathbf{S}_{p}^{(k)}\right)^{*}\right\} \\
& =\sum_{p=1}^{P} 2 \operatorname{Re}\left\{\left(\mathbf{S}_{p}^{(k)}\right)^{\mathrm{H}} \boldsymbol{E}_{n} \boldsymbol{E}_{n}^{\mathrm{H}}\left[j \boldsymbol{A}_{p}^{(k)}\right] \odot\left(\mathbf{S}_{p}^{(k)}\right)+\left(\boldsymbol{A}^{(k)}\right)^{\mathrm{T}} j\left[\boldsymbol{E}_{n} \boldsymbol{E}_{n}^{\mathrm{H}} \mathbf{S}_{p}^{(k)}\right] \odot\left(\mathbf{S}_{p}^{(k)}\right)^{*}\right\} \\
& =\sum_{p=1}^{P} 2 \operatorname{Re}\left\{\left(\mathbf{S}_{p}^{(k)}\right)^{\mathrm{H}} \boldsymbol{E}_{n} \boldsymbol{E}_{n}^{\mathrm{H}}\left[j \boldsymbol{A}_{p}^{(k)}\right] \odot\left(\mathbf{S}_{p}^{(k)}\right)+\left[\boldsymbol{E}_{n} \boldsymbol{E}_{n}^{\mathrm{H}} \mathbf{S}_{p}^{(k)}\right]^{\mathrm{T}}\left[j \boldsymbol{A}_{p}^{(k)}\right] \odot\left(\mathbf{S}_{p}^{(k)}\right)^{*}\right\} \\
& =\sum_{p=1}^{P} 2 \operatorname{Re}\left\{\left(\mathbf{S}_{p}^{(k)}\right)^{\mathrm{H}} \boldsymbol{E}_{n} \boldsymbol{E}_{n}^{\mathrm{H}}\left[j \boldsymbol{A}_{p}^{(k)}\right] \odot\left(\mathbf{S}_{p}^{(k)}\right)+\left\{\left(\mathbf{S}_{p}^{(k)}\right)^{\mathrm{H}}\left[\boldsymbol{E}_{n} \boldsymbol{E}_{n}^{\mathrm{H}}\right]^{\mathrm{H}}\left[-j \boldsymbol{A}_{p}^{(k)}\right] \odot\left(\mathbf{S}_{p}^{(k)}\right)\right\}^{*}\right\} \\
& =\sum_{p=1}^{P} 2 \operatorname{Re}\left\{\left(\mathbf{S}_{p}^{(k)}\right)^{\mathrm{H}} \boldsymbol{E}_{n} \boldsymbol{E}_{n}^{\mathrm{H}}\left[j \boldsymbol{A}_{p}^{(k)}\right] \odot\left(\mathbf{S}_{p}^{(k)}\right)-\left\{\left(\mathbf{S}_{p}^{(k)}\right)^{\mathrm{H}}\left[\boldsymbol{E}_{n} \boldsymbol{E}_{n}^{\mathrm{H}}\right]^{\mathrm{H}}\left[j \boldsymbol{A}_{p}^{(k)}\right] \odot\left(\mathbf{S}_{p}^{(k)}\right)\right\}^{*}\right\} \\
& =\sum_{p=1}^{P} 2 \operatorname{Re}\left\{2 j \operatorname{Im}\left\{\left(\mathbf{S}_{p}^{(k)}\right)^{\mathrm{H}} \boldsymbol{E}_{n} \boldsymbol{E}_{n}^{\mathrm{H}}\left[j \boldsymbol{A}_{p}^{(k)}\right] \odot\left(\mathbf{S}_{p}^{(k)}\right)\right\}\right\}=0 .
\end{array}
$$

Hence, the result given by the left-hand side of (27) is always equal to zero, i.e.

$$
2 \operatorname{Re}\left\{\left(\mathbf{S}^{(k)}\right)^{\mathrm{H}} \mathbf{W}\left[j \boldsymbol{A}^{(k)}\right] \odot\left(\mathbf{S}^{(k)}\right)\right\}+\left(\boldsymbol{A}^{(k)}\right)^{\mathrm{T}} 2 \operatorname{Re}\left\{j\left[\mathbf{W} \mathbf{S}^{(k)}\right] \odot\left(\mathbf{S}^{(k)}\right)^{*}\right\}=0 .
$$

Consequently, we obtain

$$
2 \operatorname{Re}\left\{\left(\mathbf{S}^{(k)}\right)^{\mathrm{H}} \mathbf{W}\left[j \boldsymbol{A}^{(k)}\right] \odot\left(\mathbf{S}^{(k)}\right)\right\}+\left(\boldsymbol{\Phi}^{(k)}-\boldsymbol{\Phi}_{d}\right)^{\mathrm{T}} \boldsymbol{A}^{(k)} \kappa \exp \left\{-\left[\left(\boldsymbol{\Phi}^{(k)}-\boldsymbol{\Phi}_{d}\right)^{\mathrm{T}}\left(\boldsymbol{\Phi}^{(k)}-\boldsymbol{\Phi}_{d}\right)\right] / 2\right\}<0 .
$$


It follows from (25) and (31) that

$$
J\left(\boldsymbol{\Phi}^{(k+1)}\right)<J\left(\boldsymbol{\Phi}^{(k)}\right) .
$$

The result shown by (32) ensures the convergence of the proposed method.

\section{Computer simulation results}

In this section, several simulation examples are presented for showing the effectiveness of the proposed method. For all simulation examples, we use a ULA with interelement spacing equal to half of the minimum wavelength of the signals with specified gain/null requirements. All simulation results presented are obtained by averaging $L=50$ independent runs with independent noise samples for each run. Moreover, the values of $\varepsilon$ and $\kappa$ used by the proposed method for all examples are 0.001 and 0.0001 , respectively, which were empirically found to be appropriate. Unless otherwise noted, all simulation results are based on a sample set of 15,000 snapshots. For each of the simulation examples, the results of using the proposed method are obtained after the iteration procedure is terminated. The stopping criterion for terminating the iteration procedure is that the norm of the gradient vector $\nabla_{\Phi} J(\Phi)$ is not greater than 0.01 .

Example 1. Here, three signal sources with (SNRs) equal to 10, 20, and $20 \mathrm{~dB}$, respectively, are impinging on the array with size $M=8$ from direction angles $0^{\circ}, 60^{\circ}$, and $80^{\circ}$, respectively. Consider the case of mainbeam constraint. Let the specified signal be the first one with $c_{1}=1$ and the others be the interference. The steering angle is set to $7^{\circ}$, i.e. the steering angle error equals $7^{\circ}$. Fig. 1 depicts the simulation results including the array beam patterns, the corresponding array output signal-to-interference-plus-noise ratio (SINR), and the array output SINR versus the SNR of the specified signal with and without utilizing the proposed method. For comparison, the results of using the diagonal loading technique of [20] and without steering angle error (i.e., the ideal case) are also shown. The output SINRs obtained by using the proposed method, the diagonal loading method, and the ideal case (beamforming with no steering angle error) are $16.42,7.15$, and $18.40 \mathrm{~dB}$, respectively. We observe from these results that the proposed method can effectively cope with the performance degradation due to the steering angle error.

Example 2. In this example, three signal sources with SNRs equal to 5, 4, and $2 \mathrm{~dB}$, respectively, are impinging on the array with size $M=8$ from direction angles $17^{\circ},-51^{\circ}$, and $69^{\circ}$, respectively. The specified signals are the first two signals with $c_{1}=c_{2}=1$ and the third one is the jammer. Let both direction angle errors $\Delta \theta_{1}$ and $\Delta \theta_{2}$ be equal to $7^{\circ}$. Fig. 2 plots the simulation results in terms of the array beam patterns, the corresponding array output SINR, and the array output SINR versus the SNR of the specified signal at $17^{\circ}$ with and without utilizing the proposed method. For comparison, the results of using the diagonal loading technique of [20] with a loading factor of 2000 (which was empirically found to be optimal), and the ideal case are also shown. The output SINRs obtained by using the proposed method, the diagonal loading, and the ideal errorless beamforming are $13.57,9.90$, and $13.58 \mathrm{~dB}$, respectively. We observe from the results that the proposed method can effectively cope with the performance degradation due to steering angle errors and provide array performance very close to that of the ideal case. From the array output SINR versus the number of snapshots, we note that the proposed method shows better capabilities against the finite sample effect. However, the ideal scenario demonstrates better performance than the proposed method as expected when the number of data snapshots is sufficiently large in this case.

Example 3. Here, we consider the case of four signals with SNRs equal to $5,6,7$, and $5 \mathrm{~dB}$, respectively, impinging on an array of size $M=15$ from direction angles $25^{\circ},-25^{\circ}, 50^{\circ}$, and $0^{\circ}$, respectively. Assume that the specified signals are the first three signals with $c_{1}=c_{2}=c_{3}=1$ and the fourth one is a jammer. Let all of the direction angle errors be $\Delta \theta_{1}=\Delta \theta_{2}=\Delta \theta_{3}=5^{\circ}$. Fig. 3 shows the simulation results in terms of the 

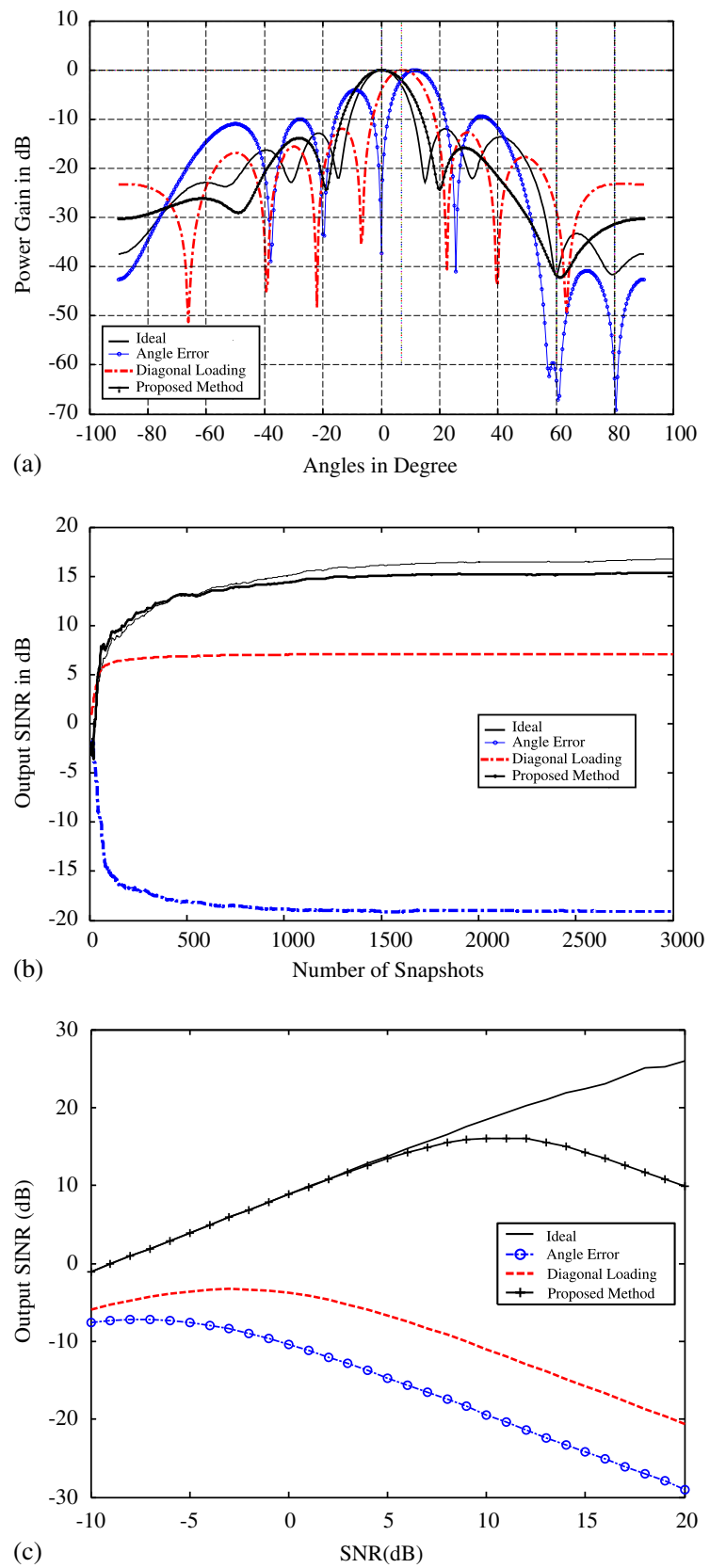

Fig. 1. (a) The array beam patterns for Example 1, (b) the output SINR versus the number of snapshots for Example 1, (c) the output SINR versus the SNR of the desired signal for Example 1.

array beam patterns using 30,000 data snapshots, the corresponding array output SINR, and the array output SINR versus the SNR of the specified signal at $25^{\circ}$ with and without utilizing the proposed method. For comparison, the results of using the diagonal loading technique of [20] with an empirically optimal 

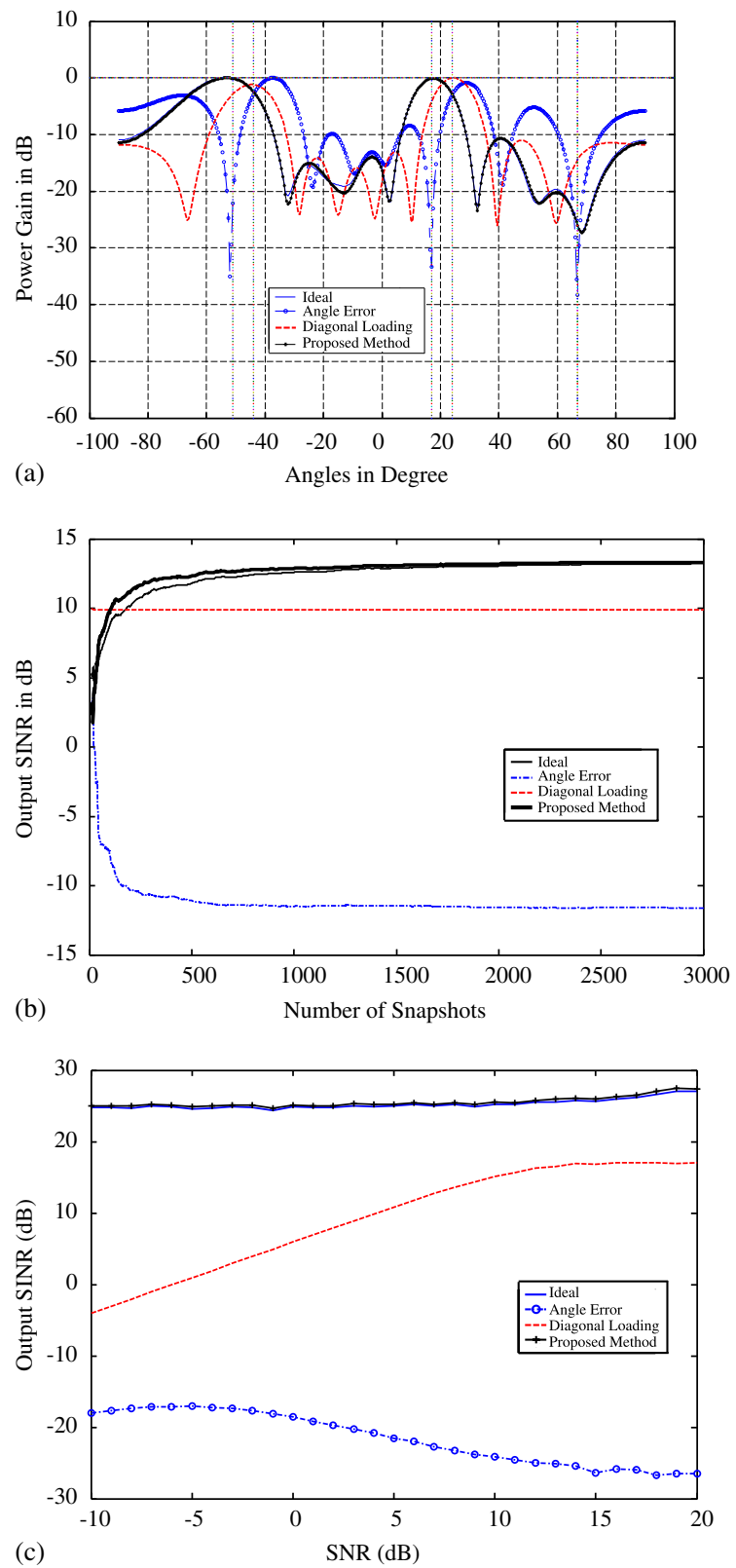

Fig. 2. (a) The array beam patterns for Example 2, (b) the output SINR versus the number of snapshots for Example 2, (c) the output SINR versus the SNR of the desired signal for Example 2.

loading factor of 2000, and the ideal beamforming case are also shown. The output SINRs obtained by utilizing 30,000 data snapshots for the results of using the proposed method, the diagonal loading, and the ideal case are 17.76, 10.87, and $17.86 \mathrm{~dB}$, respectively. Again, we observe from the simulation results that the proposed method performs very satisfactorily in the presence of steering angle errors for the multiple- 

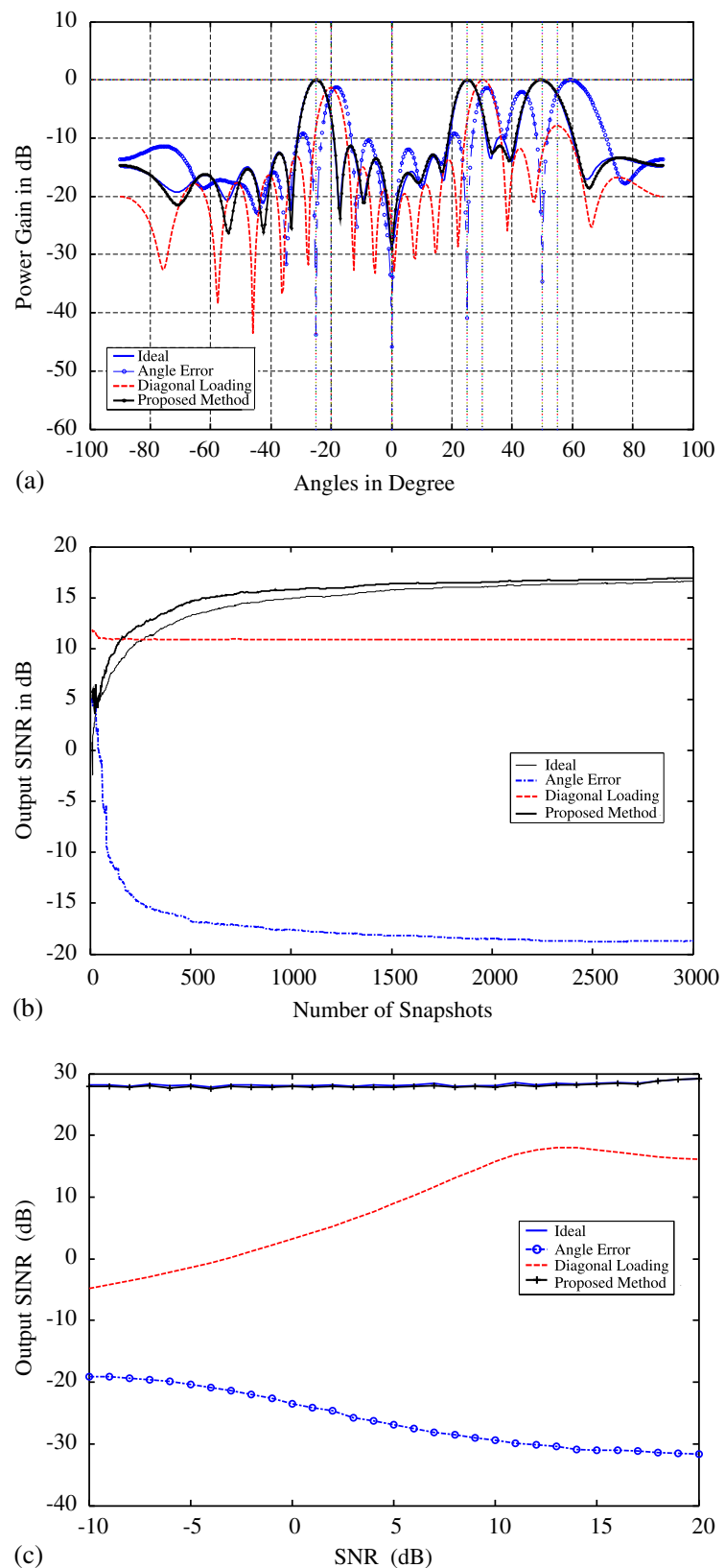

Fig. 3. (a) The array beam patterns for Example 3, (b) the output SINR versus the number of snapshots for Example 3, (c) the output SINR versus the SNR of the desired signal for Example 3.

beam case. From the array output SINR versus the number of snapshots, we note that the proposed method shows better capabilities against the finite sample effect. However, the ideal scenario demonstrates better performance than the proposed method as expected when the number of data snapshots is sufficiently large in this case. 

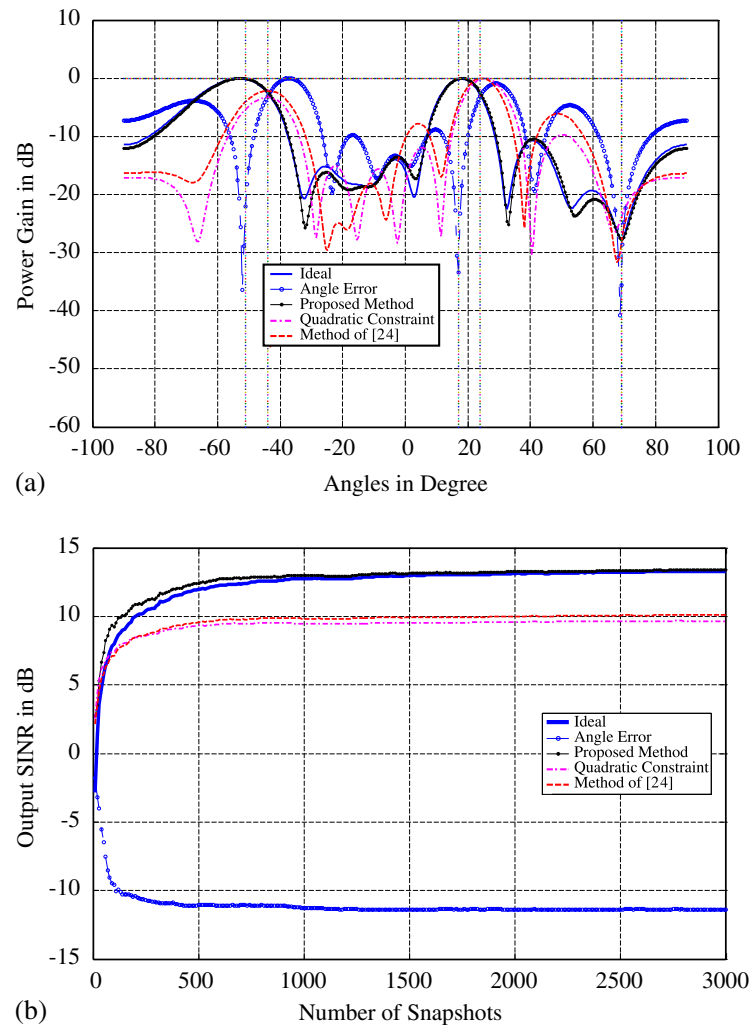

Fig. 4. (a) The array beam patterns with different constraints for Example 2, (b) the SINR versus number of snapshots under different constraints for Example 2.
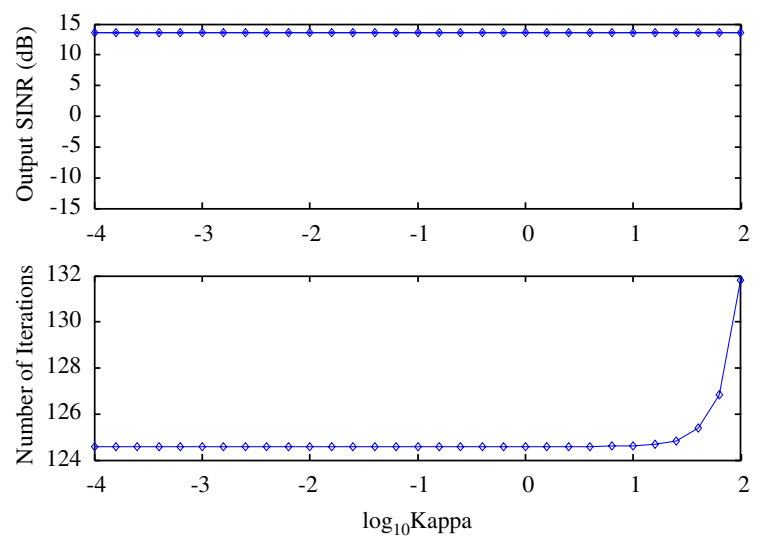

Fig. 5. The output SINR and number of iterations versus the relative weight for Example 2 .

In order to better highlight the contribution, the comparison between the proposed method, the method of [24], and using the quadratic constraint $\kappa \sum_{p=1}^{P}\left[\left(\boldsymbol{s}_{p}-\boldsymbol{a}_{d}\left(\theta_{p}\right)\right)^{\mathrm{T}}\left(\boldsymbol{s}_{p}-\boldsymbol{a}_{d}\left(\theta_{p}\right)\right)\right]$ to replace the exponential constraint $-\kappa \exp \left\{-\sum_{p=1}^{P}\left[\left(\boldsymbol{\Theta}_{s p}-\boldsymbol{\Theta}_{d p}\right)^{\mathrm{T}}\left(\boldsymbol{\Theta}_{s p}-\boldsymbol{\Theta}_{d p}\right)\right] / 2\right\}$ is also performed in terms of array beam patterns 

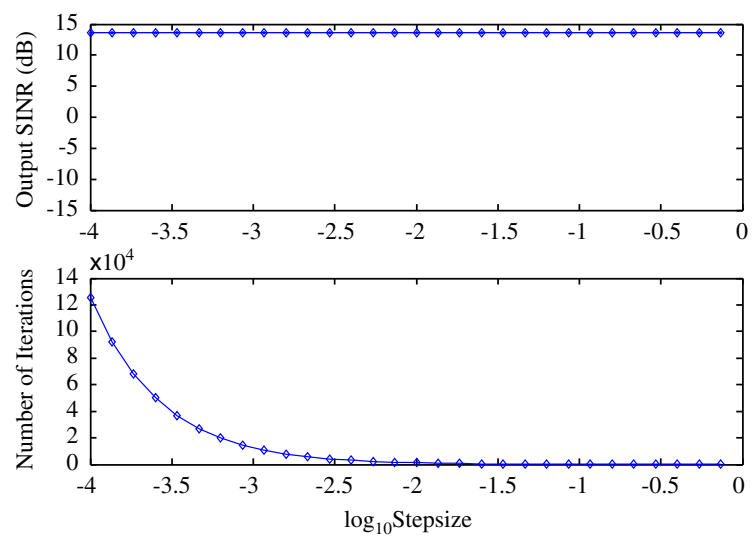

Fig. 6. The output SINR and number of iterations versus the step size for Example 2.

and output SINR versus the number of snapshots in the case of Example 2 as shown by Fig. 4. The output SINRs obtained by using the method of [24] and the quadratic constraint after using 15,000 data snapshots are 10.18 and $9.70 \mathrm{~dB}$, respectively, which are significantly less than that $(13.57 \mathrm{~dB})$ obtained by using the proposed method. These results show that the proposed method indeed outperforms the method of [24] and the method using a quadratic constraint. Finally, we present figures to illustrate how sensitive the array performance is with respect to the values of $\kappa$ and $\varepsilon$. Figs. 5 and 6 show the array output SINRs and the numbers of iterations versus $\kappa$ and $\varepsilon$, respectively, for Example 2. We note that the proposed method provides array performance with robust capabilities not very sensitive to the choice of $\kappa$ and $\varepsilon$ in the ranges shown by the figures. However, the number of iterations for obtaining the convergent results decreases in general as the value of $\varepsilon$ increases.

\section{Conclusion}

This paper has presented an efficient method for multiple-beam adaptive beamforming in the presence of steering angle errors. We have illustrated that the performance degradation of an adaptive beamformer with multiple-beam constraints due to steering angle errors is significant. The proposed method constructs a cost function consisting of the squared norm of the projection of the steering vector on the noise subspace and a constraint related to an exponential function of the squared norm of the corresponding phase error vector. The resulting minimization problem is highly nonlinear but can be solved through the use of an iterative procedure. In conjunction with a steepest-descent algorithm, the phase angle estimates for all of the signals with specified gain constraints can be obtained simultaneously. The convergence property of the proposed method has been investigated. Several simulation examples have shown the effectiveness of the proposed method in dealing with adaptive beamforming under steering angle errors.

\section{References}

[1] A.M. Vural, Effects of perturbation on the performance of optimum adaptive arrays, IEEE Trans. Aerospace Electron. Syst. 15 (February 1979) 76-87.

[2] R.T. Compton Jr., Pointing accuracy and dynamic range in a steered beam adaptive array, IEEE Trans. Aerospace Electron. Syst. 16 (May 1980) 280-287. 
[3] R.T. Compton Jr., The effect of random steering vector errors in the Applebaum adaptive array, IEEE Trans. Aerospcae Electron. Syst. 18 (September 1982) 292-400.

[4] L.C. Godara, Error analysis of the optimal antenna array processor, IEEE Trans. Aerospace Electron. Syst. 22 (May 1986) 395-409.

[5] N.K. Jablon, Adaptive beamforming with the generalized sidelobe canceller in the presence of array imperfections, IEEE Trans. Antennas Propagat. 34 (August 1986) 996-1012.

[6] K.M. Ahmed, R.J. Evans, Robust signal and array processing, IEE Proceedings Partest F, Communications in Radar and Signal Processing, August. 1982, pp. 297-302.

[7] R.J. Evans, K.M. Ahmed, Robust adaptive array antennas, J. Acoust. Soc. Amer. 71 (February 1982) $384-394$.

[8] M.H. Er, A. Cantoni, An alternative formulation for an optimum beamformer with robust capability, IEE Proceedings Port. F, Communications in Radar and Signal Process, October 1985, pp. 447-460.

[9] M.H. Er, A robust formulation for an optimum beamformer subject to amplitude and phase perturbation, Signal Processing 19 (January 1990) 17-26.

[10] M.H. Er, A. Cantoni, Derivative constraints for broad-band element space antenna array processors, IEEE Trans. Acoust. Speech Signal Process. 31 (December 1983) 1378-1393.

[11] K.M. Buckley, L.J. Griffiths, An adaptive generalized sidelobe canceller with derivative constraints, IEEE Trans. Antennas Propagat. 34 (March 1986) 311-319.

[12] H. Cox, R.M. Zeskind, M.M. Owen, Robust adaptive beamforming, IEEE Trans. Acoust. Speech Signal Processing 35 (October 1987) 1365-1376.

[13] K. Takao, N. Kikuma, Tamed adaptive antenna array, IEEE Trans. Aerospace Electron. Syst. 22 (March 1986) $388-394$.

[14] J.W. Kim, C.K. Un, A robust adaptive array based on signal subspace approach, IEEE Trans. Signal Process. 41 (November 1993) 3166-3171.

[15] A. Cantoni, X.G. Lin, K.L. Teo, A new approach to the optimization of robust antenna array processors, IEEE Trans. Antennas Propagat. 41 (April 1993) 403-411.

[16] S. Shahbazpanahi, A.B. Gershman, Z.-Q. Luo, K.M. Wong, Robust adaptive beamforming for general-rank signal models using worst-case performance optimization, Proceedings of the Second IEEE Workshop on Sonar Array and Multichannel Processing (SAM-2002), Washington DC, August 2002, pp. 13-17.

[17] S.A. Vorobyov, A.B. Gershman, Z.-Q. Luo, Robust adaptive beamforming using worst-case performance optimization: a solution to the signal mismatch problem, IEEE Trans. Signal Processing 51 (February 2003) 313-324.

[18] B.D. Carlson, Covariance matrix estimation errors and diagonal loading in adaptive arrays, IEEE Trans. Aerospace Electron. Syst. 24 (July 1988) 397-401.

[19] D.D. Feldman, L.J. Griffiths, A projection approach to robust adaptive beamforming, IEEE Trans. Signal Process. 42 (April 1994) 867-876.

[20] J. Li, P. Stoica, Z. Wang, On robust Capon beamforming and diagonal loading, IEEE Trans. Signal Process. 51 (July 2003) $1702-1715$.

[21] J.T. Mayhan, Area coverage adaptive nulling from geosynchronous satellites: phased arrays versus multiple-beam antennas, IEEE Trans. Antennas Propagat. 34 (March 1986) 410-419.

[22] K.-B. Yu, Adaptive beamforming for satellite communication with selective earth coverage and jammer nulling capability, IEEE Trans. Signal processing, 44 (December 1996) 3162-3166.

[23] J.-H. Lee, T.-F. Hsu, Adaptive beamforming with multiple-beam constraints in the presence of coherent jammers, Signal Processing 80 (November 2000) 2475-2480.

[24] C.-C. Lee, J.-H. Lee, Robust adaptive array beamforming under steering vector errors, IEEE Trans. Antennas and Propag. 45 (January 1997) 168-175.

[25] L. Chang, C.-C. Yeh, Performance of DMI and eigenspace-based beamformers, IEEE Trans. on Antennas and Propagat. 40 (November 1992) 1336-1347.

[26] R.A. Horn, C.R. Johnson, Matrix Analysis, Cambridge University Press, Cambridge, 1985.

[27] M. Wax, T. Kailath, Detection of signals by information theoretic criteria, IEEE Trans. Acoust. Speech Signal Process. ASSP-33 (February 1985) 387-392.

[28] E. Fishler, M. Grosmann, H. Messer, Detection of signals by information theoretic criteria: general asymptotic performance analysis, IEEE Trans. Acoust. Signal Process. 50 (May 2002) 1027-1036.

[29] R.F. Brcich, A.M. Zoubir, P. Pelin, Detection of sources using bootstrap techniques, IEEE Trans. Signal Process. 50 (February 2002) 206-215. 